\title{
GELEITWORT DES HERAUSGEBERS
}

Für die Sammlung „Arbeitsmethoden der modernen Naturwissenschaften" war von Anfang an auch ein Büchlein vorgesehen, das eine Anleitung zur mathematischen Behandlung naturwissenschaftlicher und insbesondere chemischer Probleme bringen sollte. Es fehlt zwar nicht an derartigen Spezialwerken. Von dem ältesten, dem allbekannten „Nernst-Schoenflies", an, den ich selbst schon als Student benutzt habe, bis zu den in den letzten Jahren erschienenen Büchern bemühen sie sich, dem Naturwissenschaftler den Gebrauch mathematischer Hilfsmittel schmackhaft zu machen, indem sie in möglichst leichtfaßlicher Form die Elemente der Differential- und Integralrechnung auseinandersetzen und Beispiele für ihre Anwendung bringen. Diese Bemühungen finden, soweit es sich um Chemiker handelt, Unterstützung durch die im Lehrplane des Chemiestudiums enthaltene Forderung des Nachweises eines Mindestmaßes an mathematischen Kenntnissen, der als Vorbedingung der Zulassung zum physikochemischen Praktikum gelten soll. Um den Studierenden der Chemie die Führung dieses Nachweises zu erleichtern, werden denn wohl auoh allenthalben mathematische Sondervorlesungen (nebst Utbungen) abgehalten, in denen die einschlägigen Kapitel aus der höheren Mathematik behandelt werden.

Es ist aber eine unter den Chemikern wohlbekannte Tatsache, daß ein großer Teil der Chemiestudierenden - von den sich speziell der physikalischen Chemie widmenden Studierenden, die von Haus aus ein engeres persönliches Verhältnis zu Physik und Mathematik haben und daher einen Sonderfall darstellen, wird hier natürlich abgesehen - die Beschäftigung mit Mathematik immer noch als einen lästigen $Z$ wang empfindet und demgemäß auch nicht leicht für eine interessierte Mitarbeit in Vorlesungen und Ubungen mathematischen Charakters zu gewinnen ist. Diese Aufgabe ist um so schwieriger zu lösen, je weniger die Studierenden den un- 
mittelbaren praktischen Nutzen dieser Disziplin für ihren ongéren chemischen Aufgabenkreis erkennen und würdigen. Wenn alss in einer solchen einführenden mathematischen Vorlesung oder Ubung die Mathematik als Selbstzweck auftritt, so ist der innere Rolitakt zwischen Lehrer und Schüler meist nur sehr schwer herzustellen. Ganz anders aber wird die Sachlage, wenn man die mathematischen Gegenstände als Hilfsmittel zur Lösung interessanter chemischer Probleme darbietet, also den pralitischen Nutzen des Vorgetragenen für das Gebiet der Chemie' in den Vordergrund stellt. Dann kann man mit relativ geringe $x$ Mühe Lust und Liebe zur Anwendung der mathematischen Behandlungsweise auch bei ursprünglich widerstrebenden Hörern erwecken und erhalten. Das Ganze ist also wesentlich ein didaktisches Problem.

Es war darum von vornherein beabsichtigt, das vorliegend Bändchen der „Arbeitsmethoden" einem Autor anzuvertrauen, der über eine ausgiebige Lehrerfahrung auf diesem Gebiete verfügt und unverkennbare Lehrerfolge aufzuweisen hat.

Einen solchen Autor haben wir erfreulicherweise in Herrn Dozenten Dr. E. Asmus in Marburg gefunden. Herr Dr. Asmus hat seit mehreren Semestern im Marburger Physikalisch-chemischen Institut die mathematischen Einführungsvorlesungen in Gestalt einer ,theoretischen Einführung in die physikalische Chemie" abgehalten und Übungen dazu durchgeführt. Seine Lehr. methode entsprach dabéi so vollkommen dem Ideal, das oben mit wenigen Strichen gezeichnet wurde, daß es ihm nicht nur gelang, das lebhafte Interesse der Gesamtheit der Chemiestudierenden für den Gegenstand zu gewinnen und bis zum Ende jeder Vorlesungsreihe zu fesseln, sondern bei seinen Hörern geradezu Freude an der Erwerbung und am gesicherten Besitze mathematischer Hilfsmittel zu erwecken, mit dem Ergebnis, daß die Hörerschaft spontan eine Fortsetzung und Vertiefung des Gebotenen in Vorlesungen und Utbungen für Fortgesohrittenere verlangte. Nichts beweist besser als diese Tatsache, daß der eingeschlagene Weg der richtige war. Denn schließlich werden Vorlesungen und Utbungen ja nicht gehalten, um nur belegt und ,mitgenommen“ zu werden, sondern zu dem Zwecke, den Hörern für Leben und Beruf Nutzen zu bringen. 
Das vorliegende Buch lehnt sich eng an die genannte Lehrmethode an. Der Leser wird ohne Mühe die grundlegenden Unterschiede gegenüber anderen Büchern mit ähnlichem Ziel erkennen. Unvermeidlich ist die Verwendung mancher Beispiele, die auch von anderen Autoren benutzt werden; das ist in der Begrenztheit des Materials an guten Beispielen begründet. Aber entscheidend - auch für den Erfolg - ist eben die Art und Weise, wie der Stoff dem Leser (oder Hörer) dargeboten wird. Hierin bringt das Buch von Asmus meines Erachtens völlig Neuartiges. Es lehrt nicht Mathematik an sich oder um ihrer selbst willen, sondern zeigt, wie man mit einem relativ bescheidenen Grundstock an mathematischem Wissen einen möglichst großen Nutzeffekt auf den vor allem den Chemiker interessierenden Gebieten erzielen kann.

Marburg, im Februar 1942.

\section{A. Thiel $\dagger$}

Nachdem der Satz dieses Buches zweimal, ehe es zum Druck kam, zerstört wurde, kann es nun endlich erscheinen und damit. der Wunsch des hochgeschätzten leider viel zu früh verstorbenen Herausgebers A. Thiel, der an diesem Werke besonders hing, erfüllt werden.

Berlin, im April 1947.

Walter de Gruyter \& Co. 